\title{
Effect on attendance at breast cancer screening of adding a self administered questionnaire to the usual invitation to breast screening in southern England
}

Emily Banks, Ann Richardson, Valerie Beral, Barbara Crossley, Moya Simmonds, Elizabeth Hilton, Ruth English, Judy Davis, Joan Austoker
Imperial Cancer Research Fund, Cancer Epidemiology Unit, Gibson Building, Radcliffe Infirmary, Oxford, OX2 6HE

E Banks

V Beral

B Crossley

M Simmonds

E Hilton

Department of Public Health and General Practice, Christchurch School of Medicine, New Zealand

A Richardson

Breast Care Unit, Oxford Radcliffe NHS

Trust, Oxford R English

West of London Breast Screening Unit, Charing Cross

Hospital, London

J Davis

Cancer Research Campaign Primary Care Education Group, Oxford

J Austoker

Correspondence to: Dr Banks.

Accepted for publication 24 May 1997

\begin{abstract}
Study objective-The primary aim of the research described in this paper was to ascertain whether inclusion of a self administered questionnaire with the usual invitation to routine breast screening affected screening uptake. Secondary aims included establishing appropriate questionnaire distribution and collection methods within the framework of the National Health Service Breast Screening Programme and optimisation of questionnaire design.

Design-Randomised study.

Setting-Oxfordshire and West of London Breast Screening Units.

Participants/Methods-6400 women invited for routine screening mammography were individually randomised to receive either the usual breast screening invitation alone, or to receive the usual invitation accompanied by a self administered questionnaire, to be returned at the time of screening. Participants were then followed up and attendance rates at screening were compared between groups. Main results-Screening attendance rates were similar in women who did and did not receive a questionnaire $(71 \%$ in each group). Of those who were sent a questionnaire and attended for screening, $77 \%$ returned a completed questionnaire. Screening uptake was not affected by the way in which the questionnaire was returned or by whether or not personal details and signed permission for follow up were requested.

Conclusions-The inclusion of a self administered questionnaire accompanying the invitation to breast screening did not adversely affect screening uptake. A high proportion of women completed the questionnaire.
\end{abstract}

(F Epidemiol Community Health 1998;52:116-119)

In the UK, all women aged 50-64 registered with a general practitioner are invited to attend the National Health Service Breast Screening Programme (NHSBSP) approximately once every three years. Each woman is sent a letter of invitation, which is despatched two to four weeks before her designated appointment for screening. In the year up to 31 March 1995, the
NHSBSP screened over 1.2 million women, representing $77 \%$ of those invited to attend. ${ }^{1}$ The large number of women screened, the centralised nature of the programme, and the high participation rates make the NHSBSP an ideal setting in which to conduct population based research into women's health. In addition, the age of women invited to screening corresponds to the time of peak hormone replacement therapy (HRT) use. ${ }^{2}{ }^{3}$ Bearing this in mind, the central aim of the NHSBSP is the reduction in breast cancer mortality through the early detection of breast cancer, ${ }^{1}$ and any research taking place within the NHSBSP must ensure that it does not compromise the effectiveness of the programme.

This article reports on the three pilot studies of a major study that aims to investigate the effects of HRT on health, through the NHSBSP. The final study will entail the distribution and collection of a self administered questionnaire accompanying the usual invitation to breast screening, requesting demographic, reproductive, health, and HRT related information. Women will then be followed up through the breast screening centres and the National Health Service Central Register. A summary of the general aims and methods of the pilot studies will be given first, followed by a more detailed description of the methods and results of each pilot study.

\section{Aims and objectives}

The main aim of the pilot studies was to ascertain whether inclusion of a self administered questionnaire with the usual invitation to routine breast screening affected screening uptake. The first two pilot studies were also designed to assess the effect of differing methods of returning the questionnaire and the effect of requesting personal details and signed permission for follow up.

\section{Methods}

\section{ACCEPTABILITY STUDY}

Before conducting the pilot studies, a preliminary investigation was carried out at the Oxford Breast Screening Unit to establish whether women who had attended for screening were willing to fill out a self completed questionnaire about their health and whether such a questionnaire raised any concerns or caused distress to the women involved. Two hundred women who had not had breast cancer diagnosed at their most recent breast 
Table 1 Pilot study screening attendance results

\begin{tabular}{|c|c|c|c|c|c|}
\hline Pilot & Location & Group definition & $\begin{array}{l}\text { Number } \\
\text { invited for } \\
\text { screening }\end{array}$ & $\begin{array}{l}\text { Number } \\
(\%) \\
\text { attending } \\
\text { screening }\end{array}$ & $\begin{array}{l}\text { Questionnaire } \\
\text { returned } \\
\text { number (\% of } \\
\text { those screened) }\end{array}$ \\
\hline \multirow[t]{3}{*}{1} & \multirow[t]{3}{*}{ Bicester (Oxon) } & No questionnaire & 435 & $348(80)$ & \\
\hline & & Questionnaire: return at appointment & 369 & $292(79)$ & $226(77)$ \\
\hline & & Questionnaire: return by post & 396 & $312(79)$ & $257(82)$ \\
\hline \multirow[t]{4}{*}{2} & \multirow{4}{*}{$\begin{array}{l}\text { Charlbury and Oxford } \\
\text { (Oxon) }\end{array}$} & No questionnaire & 396 & $316(80)$ & \\
\hline & & Questionnaire: no signature or details & 404 & $295(73)$ & $235(80)$ \\
\hline & & Questionnaire: signature and details & 197 & $143(73)$ & $113(79)$ \\
\hline & & $\begin{array}{l}\text { Questionnaire: signature, details, town of birth, } \\
\text { surname at birth }\end{array}$ & 203 & $164(81)$ & $131(80)$ \\
\hline \multirow[t]{2}{*}{3} & \multirow[t]{2}{*}{ Banbury (Oxon) } & No questionnaire & 981 & $744(76)$ & \\
\hline & & Questionnaire & 1019 & $768(75)$ & $601(78)$ \\
\hline \multirow[t]{4}{*}{3} & \multirow[t]{2}{*}{ Ealing (London) } & No questionnaire & 1000 & $577(58)$ & \\
\hline & & Questionnaire & 1000 & $568(57)$ & $381(67)$ \\
\hline & \multirow[t]{2}{*}{ Total } & No questionnaire & 2812 & $1985(71)$ & \\
\hline & & Questionnaire & 3588 & $2542(71)$ & $1944(77)$ \\
\hline
\end{tabular}

screening visit were randomly selected to participate. These women were sent a covering letter explaining the purpose of the survey, a postal questionnaire, and a colour photograph of the commonly prescribed types of HRT, together with a postage paid envelope for returning the questionnaire. Women were asked to complete the questionnaire. In addition they were asked to comment on the survey, including any questions they may have and if they would provide their telephone number so that aspects of the survey could be checked if necessary. A reminder letter was sent to those women not returning the questionnaire within two weeks. One hundred and fifty two questionnaires were received, giving a response rate of $76 \%$. A member of the NHSBSP Advisory Committee (JA) telephoned a random sample of 50 women after they had returned the questionnaire, to ask how they felt about the content of the questionnaire, and in particular whether any aspect of the questionnaire was worrying to them. No women expressed any concerns about the content of the questionnaire or about having received it. It was therefore concluded that the pilot studies should be allowed to proceed.

PILOT STUDY METHODS

From November 1994 to April 1996 a total of 6400 women invited to routine breast screening at the Oxfordshire Breast Screening Unit and West of London Breast Screening Service participated in three pilot studies. All women included in the pilot studies were randomised to receive or not to receive the questionnaire and in the first pilot study (involving 1200 women) women were also randomised by the method by which the questionnaire was to be returned and the second (involving 1200 women) also randomised by type of personal details requested.

For each pilot study a commencement date was chosen and all women due to be sent routine invitations to screening from that date onwards were individually randomised until the required sample size was achieved. There were no exclusion criteria. Women randomised to no intervention received the usual invitation to breast screening. Women randomised to the questionnaire group received a self administered questionnaire accompanying their invitation to breast screening, with instructions on how it should be returned. Each questionnaire was labelled with the woman's unique breast screening identification number. The time between the invitation and the appointment for breast screening was approximately three weeks. Attendance at screening was then ascertained for each woman using the screening centres' computerised records and the breast screening identification number.

As some women changed their appointment and attended screening at a later date, attendance at screening was recorded for up to three months after the last scheduled appointment date for the women recruited in that study.

STATISTICAL METHODS

Individual randomisation for the Oxfordshire based pilot studies used random numbers generated by NAG routines. The West of London pilot study used a repeated string of six random numbers generated by the same programme. Data recorded on the questionnaire were double entered into the EpiInfo database, and were analysed using EpiInfo version 5. Basic $\chi^{2}$ tests were used to assess whether there was an association between receiving a questionnaire and attending for screening. Where appropriate the Mantel-Haenszel method was used to stratify by screening centre. Results with a $p$ value of less than 0.05 were considered statistically significant. As for most of the pilot studies only women who attended for screening were able to return the questionnaire, the response rate to the questionnaire was calculated only among screened women.

First pilot study: randomisation by questionnaire and by method of questionnaire return

During November and December 1994, 1200 women due to attend the Oxfordshire mobile breast screening unit at Bicester were individually randomised into three groups. The 435 women in the first group did not have the study intervention and received only the usual invitation to breast screening. The second group consisted of 369 women who were sent the study questionnaire along with the invitation to screening and were asked to bring the completed questionnaire with them when they attended their screening appointment. The 396 women in the third group received the 
questionnaire with their invitation, along with a prepaid envelope and a request to return the completed questionnaire by post. Screening attendance was followed up until March 1995 using the methods outlined above.

Overall, there was no significant difference in screening attendance between the three randomised groups; $80 \%, 79 \%$, and $79 \%$ respectively (table 1). A total of 503 questionnaires were received, including 20 questionnaires from women who did not attend screening. Among those attending for screening, there was no significant difference in the proportion who returned a completed questionnaire, whether they were asked to bring it with them to screening $(77 \%)$ or to return the questionnaire by post $(82 \%)(\mathrm{p}=0.13)$.

It was concluded that there was no difference in screening attendance for each group. Although slightly more questionnaires were received in the group returning the questionnaire by post, this method was not deemed to be cost effective (see Discussion). It was decided that in future women would be asked to bring the completed questionnaire with them when they attended for screening.

\section{Second pilot study: randomisation by questionnaire and by request for follow up}

This study was conducted at the Oxfordshire breast screening service, among women invited to attend for breast screening at the Churchill hospital and at the mobile breast screening unit in Charlbury from May to July 1995. A total of 1200 women were randomised into three groups, with the third group being further randomised into two. The first group of 396 women received the usual letter of invitation to screening. The second group contained 404 women who received a questionnaire together with their usual invitation to screening, but the questionnaire did not ask for any personal details or for signed permission for follow up. The third group was further randomised into group 3.1 (197 women), who received a questionnaire that included a request for personal details and signed permission for follow up and group 3.2 (203 women), who received the same questionnaire as those in group 3.1, with additional questions regarding their surname at birth and the town where they were born. All women who received a questionnaire were asked to bring the completed questionnaire with them when they attended their screening appointment. Attendance was followed up until October 1995.

Overall, 918 of $1200(77 \%)$ women attended screening. Attendance varied significantly between those invited to screening at the Churchill hospital (66\% attendance) and those invited to attend the mobile unit in Charlbury $(82 \%$ attendance $)(\mathrm{p}<0.0001)$. Analyses were therefore stratified by screening location. No significant difference in screening attendance was seen according to whether or not a questionnaire was received by the woman, or by the type of questionnaire received, stratified by screening location. Questionnaires were
KEY POINTS

- The questionnaire requested personal information regarding lifestyle, past and current health, reproductive factors, family history of breast cancer, and hormone replacement therapy.

- The response rate to the questionnaire was high.

- Including this questionnaire with the routine invitation to breast screening did not affect screening attendance.

returned by $80 \%$ of women who attended for screening and had been sent a questionnaire with their invitation (table 1).

It was concluded that attendance was not compromised by the request for personal details and signed permission for follow up. It was therefore decided that the questionnaire should request comprehensive personal details, including surname at birth and the town where the subject was born, to aid in identification of the subject at follow up. From this stage onward signed permission for follow up was also requested.

\section{Third pilot study: randomisation by} questionnaire alone

From October to December 1995, 2000 women invited to attend Oxfordshire mobile breast screening unit in Banbury were randomised into two groups: 981 received no intervention and 1019 received a self administered questionnaire. From February to April 1996 a further 2000 women who were invited to attend the West of London mobile breast screening unit in Ealing were randomised into the same groups: 1000 receiving no intervention and 1000 receiving the questionnaire. The questionnaire was the final version that was to be used in the main study and it requested signed permission for follow up and was sent out with the routine invitation to screening. Women were asked to return the completed questionnaire when they attended their breast screening appointment. Follow up was completed in July 1996.

The total attendance rate at screening was $75 \%$ for those invited to screening at Banbury and $57 \%$ for those invited to screening at Ealing. No significant difference was seen among those receiving the questionnaire and those in the non-intervention group, at either location $(76 \%$ versus $75 \%$ in Banbury and $58 \%$ versus $57 \%$ in Ealing). At the Banbury mobile screening unit $78 \%$ of those issued with questionnaires and attending screening returned their questionnaires, and at the Ealing mobile $67 \%$ returned the questionnaire (table $1)$.

\section{Discussion}

A total of 6400 women were randomised in these pilot studies; 3588 received a questionnaire with their usual invitation to breast screening; 2812 just received their usual letter of invitation. A total of 2542 women in the questionnaire group attended screening, as did 
1985 of those not receiving a questionnaire. Stratifying by screening location, no significant difference in attendance at breast screening was seen between those receiving the questionnaire and those who had no intervention $(\mathrm{p}=0.2)$. Nor were there differences in attendance according to how the questionnaire was returned, the type of personal details requested or whether or not signed permission for follow up was requested. The same result was seen in both urban and rural settings. Overall, $71 \%$ of women sent a questionnaire attended for screening and of these, $77 \%$ returned a completed questionnaire (table 1 ).

A previous study into the effect of research on attendance at breast screening was conducted in the Netherlands in mid 1992. ${ }^{4}$ The authors compared breast screening attendance in 1863 women invited to participate in the European Prospective Investigation of Cancer (EPIC) study, with attendance among an equal number of control women. Although the EPIC study was time consuming and involved taking a blood sample, the authors found no difference in breast screening attendance between the study group and their controls, in the age group 50-69. A difference in attendance was noted in women over age 69 , but these women were subsequently not included in the screening programme. They concluded that inviting women to be involved in an additional scientific study does not deter them from attending breast screening. ${ }^{4}$

In the pilot studies described here, screening attendance did not vary according to the type of questionnaire return. In addition, only those women attending breast screening can be followed up for breast cancer through the screening service, so postal questionnaires received from women not attending screening cannot be used in this part of the study. Identifying women who had returned a questionnaire but had not attended screening would also be difficult if all participants returned their questionnaires by post. For these reasons, asking the subject to bring her completed questionnaire with her to her breast screening appointment was ultimately considered to be the best method of returning the questionnaire.

In conclusion, these pilot studies show that including a self administered questionnaire with the usual invitation to screening does not affect breast cancer screening attendance.

We thank all of the radiographers and administrative staff at the breast screening centres for their cooperation, and we are grateful to Janet Green and Denise Scrivener for help with the distribution and collection of questionnaires. We also thank Nicola Fear and Hollie Thomas for their assistance.

Funding: this research was supported by the Imperial Cancer Research Fund.

Conflicts of interest: none.

1 Patnick J, ed. NHS Breast Screening Programme Review 1996. Sheffield: NHSBSP, 1996.

2 Isaacs $\mathrm{AJ}$, Britton $\mathrm{AR}$, McPherson $\mathrm{K}$. Utilisation of Isacs AJ, Britton AR, McPherson $\mathrm{K}$. Utilisation of
hormone replacement therapy by women doctors. BMF 1995;311:1399-401.

3 Banks E, Crossley B, English R, Richardson A. Women doctors' use of hormone replacement therapy: High prevalence of use is not confined to women doctors. BMF 1996; 312:638.

4 Peeters PH, Beckers CG, Hogervorst JM, Collette HJ. Effect on breast cancer screening response in The Netherlands of inviting women for an additional scientific investigation. F Epidemiol Community Health 1994;48:175-7. 